\title{
BMJ Open Cost-effectiveness of treatment optimisation with biomarkers for immunotherapy in solid tumours: a systematic review protocol
}

\author{
Sara Mucherino (D) , ${ }^{1}$ Valentina Lorenzoni, ${ }^{2}$ Valentina Orlando, ${ }^{1}$ Isotta Triulzi, ${ }^{2}$ \\ Marzia Del Re, ${ }^{3}$ Annalisa Capuano, ${ }^{4}$ Romano Danesi, ${ }^{3}$ Giuseppe Turchetti, ${ }^{2}$ \\ Enrica Menditto ${ }^{1}$
}

To cite: Mucherino S, Lorenzoni V, Orlando V, et al. Cost-effectiveness of treatment optimisation with biomarkers for immunotherapy in solid tumours: a systematic review protocol. BMJ Open 2021;11:e048141. doi:10.1136/ bmjopen-2020-048141

- Prepublication history and additional supplemental material for this paper are available online. To view these files, please visit the journal online (http://dx.doi.org/10.1136/ bmjopen-2020-048141).

Received 17 December 2020 Accepted 30 July 2021

Check for updates

(C) Author(s) (or their employer(s)) 2021. Re-use permitted under CC BY-NC. No commercial re-use. See rights and permissions. Published by BMJ.

For numbered affiliations see end of article.

Correspondence to Dr Enrica Menditto; enrica.menditto@unina.it

\section{ABSTRACT}

Introduction The combination of biomarkers and drugs is the subject of growing interest both from regulators, physicians and companies. This study protocol of a systematic review is aimed to describe available literature evidences about the cost-effectiveness, cost-utility or netmonetary benefit of the use of biomarkers in solid tumour as tools for customising immunotherapy to identify what further research needs.

Methods and analysis A systematic review of the literature will be carried out according to the Preferred Reporting Items for Systematic Reviews and MetaAnalyses statement guidelines. PubMed and Embase will be queried from June 2010 to June 2021. The PICOS model will be applied: target population $(\mathrm{P})$ will be patients with solid tumours treated with immune checkpoint inhibitors (ICls); the interventions (I) will be test of the immune checkpoint predictive biomarkers; the comparator (C) will be any other targeted or non-targeted therapy; outcomes (0) evaluated will be health economic and clinical implications assessed in terms of incremental cost-effectiveness ratio, net health benefit, net monetary benefit, life years gained, quality of life, etc; study (S) considered will be economic evaluations reporting costeffectiveness analysis, cost-utility analysis, net-monetary benefit. The quality of the evidence will be graded according to Grading of Recommendations Assessment, Development and Evaluation.

Ethics and dissemination This systematic review will assess the cost-effectiveness implications of using biomarkers in the immunotherapy with ICls, which may help to understand whether this approach is widespread in real clinical practice. This research is exempt from ethics approval because the work is carried out on published documents. We will disseminate this protocol in a related peer-reviewed journal.

PROSPERO registration number CRD42020201549.

\section{INTRODUCTION}

In recent years, the pharmaceutical industry has seen a shift from the blockbuster model, in which drugs are developed for an ideal patient, to a nichebuster model, in which drugs are developed specifically for specific
Strengths and limitations of this study

- The use of predictive biomarkers in the therapy with immune checkpoint inhibitors can help target therapy in some solid tumours, hence, the combination of biomarkers and drugs is the subject of growing interest both from regulators, physicians and companies.

- This is the first systematic review which will specifically describe available literature evidences about the cost-effectiveness, cost-utility or net-monetary benefit of the use of biomarkers in solid tumour as tools for customising immunotherapy to identify what further research needs.

- An in-depth search strategy will be applied to two major scientific databases, without geographical and conducted by a multidisciplinary team with expertise in the field.

- The quality of studies included and related level of evidence will be assessed quality using the Consolidated Health Economic Evaluation Reporting Standard checklist and the Grading of Recommendations Assessment, Development and Evaluation tool.

patient groups. ${ }^{12}$ In this context, the combination of biomarkers and drugs is the subject of growing interest both from regulators, physicians and companies. $^{3-7}$

The US Food and Drug Administration (FDA) regularly publishes and updates a list of drugs for which it is suggested or mandatory to associate a genetic-molecular test. ${ }^{8}$ The importance of predictive biomarkers is related to optimising patient benefits, reducing the risk of toxicity and leading combined approaches. ${ }^{9}$ Particularly, for some drugs the test result defines whether or not to administer, for others it establishes the most appropriate dosage of therapy. Among the 166 biomarker-drug combinations reported by the FDA, in only $29 \%$ (48 combinations) 
of cases, results obtained from biomarker test have an impact on the physician's choice to prescribe or not prescribe a particular drug. ${ }^{10}$ In Italy, 34 of those 48 combinations are approved for use and among these, about $80 \%$ find application in oncology particularly for solid tumours treatment. ${ }^{10}$ The clinical development of checkpoint inhibitor-based immunotherapy has ushered in an exciting era of anticancer therapy. Since the FDA approval of ipilimumab (human IgG1 k anti-CTLA-4 monoclonal antibody) in 2011, six more immune checkpoint inhibitors (ICIs) have been approved for cancer therapy. Programmed death-1 (PD-1) inhibitors nivolumab, pembrolizumab, cemiplimab and programmed death ligand-1 (PD-L1) inhibitors atezolizumab, avelumab and durvalumab are in the current list of the approved agents in addition to ipilimumab. ${ }^{11}$ The importance of predictive biomarkers is related to the optimisation of benefits in patients treated with immunotherapy, by reducing the risk of toxicity and leading combined approaches. Durable responses have been observed in patients with various malignant neoplasms. ${ }^{12}$

This study protocol is part of a funded Italian National Research Project based on the hypothesis that the identification of predictive biomarkers can improve the understanding of the mechanisms underlying the complex interactions between the immune system and cancer thus guiding clinicians to optimise therapy with monoclonal anti-PD-1 and anti-PD-L1 antibodies. Hence, among the already known biomarkers, the overexpression of PD-L1 is an important and widely explored predictive biomarker for the response to PD-1/PD-L1 antibodies. ${ }^{43}$ Direct assessment of PD-L1 expression on tumour cells is a logical biomarker for the prediction of treatment response to anti-PD-1 or anti-PD-L1 therapies. ${ }^{14} 15$ The use of PD-1 and PD-L1 as predictive biomarkers can help target therapy in some solid tumours, including renal and non-small cell lung cancer (NSCLC) ${ }^{16-18}$ Nivolumab and pembrolizumab and an PD-L1 inhibitor, atezolizumab, have also been approved by the Italian Medicines Agency (AIFA), for the treatment of patients with NSCLC. ${ }^{19-21}$

A targeted approach to treatment using predictive biomarkers has the potential not only to maximise clinical benefit in respect to not-targeted therapy, but also to improve cost-effectiveness and reduce the economic burden of the disease..$^{22}$ As the global impact of these types of cancers continues to grow, the implementation of new and more effective therapies becomes important but also overly expensive. ${ }^{23}$ Therefore, the analysis of the health economics implications of the use of biomarkers upstream of the choice of the specific therapy represents an imperative to validate its effectiveness, the eventual relationship with the quality of life, patient reported outcomes and sustainability. ${ }^{24}$ However, there is no existing peerreviewed or published synthesis summarising the impact of predictive biomarkers use in oncological treatment in health economics terms.

This is the protocol of a systematic review aimed at describing available literature about the cost-effectiveness, cost-utility or net-monetary benefit of the use of predictive biomarkers in solid tumour treated with ICIs as tools for customising immunotherapy; the final goal of the study is to help decision-makers and clinicians identify the most effective and sustainable options and highlight further research needs.

\section{METHODS AND ANALYSIS}

The Preferred Reporting Items for Systematic Review and Meta-Analysis Protocols (PRISMA-P) 2015 checklist was used to develop the present study protocol. Modifications in the item sequencies were done where appropriate. $^{25}$

\section{Information sources}

A systematic review of the literature will be carried out according to the PRISMA 2020 statement guidelines. ${ }^{26}$ For the present review, the identification of relevant studies will be achieved by searching electronic databases of the published literature. In details Medical Literature Analysis and Retrieval System Online (via PubMed/ MEDLINE) and Embase (via Ovid) were queried from June 2010 to June 2021.

\section{Search strategy}

The search strategy will be developed and completed in PubMed, and then the same strategy will be applied to Embase. The search strategy was developed according to the PICOS model and based on the existing literature and finally revised by clinicians. More in detail, the search strategy will combine headings and keywords listed in table 1 answering each questions of the PICOS model. Those terms combined with boolean operators AND/OR will be searched both as Medical Subject Headings (MeSH) term (PubMed) or Emtree (Embase) both in title and abstract. The full-search strategy that will be used is reported in table 1 . More in detail, the search syntax for the two databases are presented in the online supplemental appendix 1 .

\section{Elegibility criteria}

The inclusion criteria are based on compliance with the PICOS. Particularly, we will identify:

- Patient (P): Patients with solid tumours treated with ICIs (monotherapy or combination therapy): nivolumab, pembrolizumab, ipilimumab, atezolizumab, durvalumab, avelumab, cemiplimab.

- Intervention (I): Test of the immune checkpoint predictive biomarkers, such as PD-1, PD-L1, CTLA-4, interleukin-6.

- Comparator (C): Any other targeted or non-targeted therapy.

- Outcomes (O): Health-economic outcomes (incremental cost-effectiveness, incremental costeffectiveness ratios, net health benefit, net monetary benefit, life years, quality adjusted life years, etc) will be evaluated between ICIs therapy. 


\begin{tabular}{|c|c|}
\hline Query & $\begin{array}{l}\text { Keywords (MeSH terms/Emtree OR Title and } \\
\text { Abstract) }\end{array}$ \\
\hline \#1 & Antibodies, monoclonal \\
\hline \#2 & Immunotherapy \\
\hline$\# 3$ & $\begin{array}{l}\text { nivolumab/ipilimumab[title/abstract] OR } \\
\text { ipilimumab[title/abstract] OR durvalumab[title/ } \\
\text { abstract] OR atezolizumab[title/abstract] OR } \\
\text { nivolumab[title/abstract] OR pembrolizumab[title/ } \\
\text { abstract] OR “Ipilimumab"[MeSH] OR } \\
\text { "durvalumab"[supplementary concept] } \\
\text { OR "avelumab"[supplementary concept] } \\
\text { OR "atezolizumab"[supplementary } \\
\text { concept] OR "Nivolumab"[MeSH] OR } \\
\text { "pembrolizumab"[supplementary concept] }\end{array}$ \\
\hline \#4 & Immune checkpoint inhibitor \\
\hline \#5 & Neoplasms \\
\hline \#6 & Cancer \\
\hline \#7 & Carcinoma \\
\hline \#8 & Tumor OR Toumor \\
\hline \#9 & Target therapy OR Chemotherapy \\
\hline \#10 & $\begin{array}{l}\text { Biomarkers OR PD-1 OR Programmed Death } 1 \\
\text { OR PD-L1 OR Programmed Death Ligand } 1 \text { OR } \\
\text { IL-6 OR Interleukin-6 OR CTLA-4 }\end{array}$ \\
\hline \#11 & Cost-benefit analysis \\
\hline \#12 & Cost-effectiveness \\
\hline \#13 & Cost-utility \\
\hline$\# 14$ & Economic evaluation \\
\hline \#15 & Quality of life \\
\hline \#16 & 1 OR 2 OR 3 OR 4 \\
\hline \#17 & 5 OR 6 OR 7 OR 8 \\
\hline \#18 & 11 OR 12 OR 13 OR 14 OR 15 \\
\hline \#19 & 16 AND 17 \\
\hline \#20 & 19 AND 9 AND 10 \\
\hline \#21 & 20 AND 18 \\
\hline
\end{tabular}

MeSH, Medical Subject Headings.

- Study design (S): Health-economic evaluations reporting cost-effectiveness analysis, cost-utility analysis, net-monetary benefit and conducted within clinical trials or observational studies.

All peer-reviewed original articles about health economics evaluation related to biomarkers use published between June 2010 to June 2021 and responding to the PICOS will be considered for inclusion in the study. On the other hand, conference proceedings, rationale and/ or study protocol, letters, editorials, commentaries, case reports, case study, case series, review, consensus, guidelines, expert opinions and grey literature will not be included (exclusion criteria).

Moreover, language restriction will be applied to the research, as fundamental to the eligibility of the study
Table 2 Synthesis of inclusion and exclusion criteria

\begin{tabular}{lll}
\hline $\begin{array}{l}\text { Selection } \\
\text { criteria }\end{array}$ & Inclusion criteria & Exclusion criteria \\
\hline $\begin{array}{l}\text { Language study } \\
\text { type }\end{array}$ & English & Non-English \\
\hline $\begin{array}{l}\text { Time limit } \\
\text { (years) }\end{array}$ & $2010-2021$ & $<2010$ \\
Study design & $\begin{array}{l}\text { Published and } \\
\text { peer-reviewed } \\
\text { health economic } \\
\text { evaluations }\end{array}$ & $\begin{array}{l}\text { Conference } \\
\text { proceedings, } \\
\text { rationale and/ } \\
\text { or design, } \\
\text { letters, editorials, } \\
\text { commentaries, } \\
\text { case reports, case } \\
\text { study, case series, } \\
\text { review, consensus } \\
\text { guidelines, expert } \\
\text { opinions, grey } \\
\text { literature }\end{array}$ \\
\hline
\end{tabular}

will be the availability of the papers' full text published in English.

Any identified literature reviews will be used as a source for finding additional articles not present in our data set.

Inclusion and exclusion criteria are summarised in table 2.

The quality of the economic evaluations that will be included in the study will be assessed through the Consolidated Health Economic Evaluation Reporting Standard (CHEERS) checklist. $^{27}$

\section{Selection and data process}

The references will be collected using the software program Reference Manager, V.12 (Institute for Scientific Information, Berkeley, California, USA). All references will be screened for relevance and, those potentially eligible will be assessed, according to the inclusion/exclusion criteria, accepted or rejected, as appropriate.

Four researchers will double screen titles and abstracts to discard irrelevant ones in the first screening phase. Then, full texts of the records selected from the previous step will be retrieved and double screened to assess the eligibility for the inclusion in the qualitative analysis. Finally, the references obtained will be validated by clinicians and researchers in the fields of pharmacology, immunotherapy, pharmacovigilance, pharmacoeconomics. Reference lists from included records will be also screened to identify additional papers (backward reference searching) as for other studies citing that paper (forward citation searching).

The type of information that will be extracted from each reference included in the qualitative analysis and collected into a dedicated file are reported in table 3 . The structure of the table that will be used to describe results obtained is shown in online supplemental appendix 2 . Changes to the variables in the table could be made in the 


\begin{tabular}{|c|c|}
\hline Data extraction & Description \\
\hline Reference & All paper identification details \\
\hline Publication year & Year of publication of the paper \\
\hline $\begin{array}{l}\text { Perspective of the } \\
\text { analysis }\end{array}$ & $\begin{array}{l}\text { National health service, society, } \\
\text { government, patient }\end{array}$ \\
\hline Type of costs & $\begin{array}{l}\text { Direct healthcare costs, direct non- } \\
\text { health costs, indirect costs, intangible } \\
\text { costs }\end{array}$ \\
\hline $\begin{array}{l}\text { Reference year of } \\
\text { costs }\end{array}$ & $\begin{array}{l}\text { Specific year of reference of costs if } \\
\text { reported }\end{array}$ \\
\hline
\end{tabular}

Patient diagnosis Each status of: breast cancer, bladder cancer, cervical cancer, colon cancer, head and neck cancer, Hodgkin's lymphoma, liver cancer, lung cancer, renal cell cancer (a type of kidney cancer), skin cancer, stomach cancer, rectal cancer and any solid tumour that is not able to repair errors in its DNA that occur when the DNA is copied.

\begin{tabular}{ll} 
Patient (P) & $\begin{array}{l}\text { Patients with solid tumours treated } \\
\text { with immune checkpoint inhibitors } \\
\text { (monotherapy or combination } \\
\text { therapy): nivolumab, pembrolizumab, } \\
\text { ipilimumab, atezolizumab, } \\
\text { durvalumab, avelumab, cemiplimab }\end{array}$ \\
& $\begin{array}{l}\text { Test of the immune checkpoint } \\
\text { predictive biomarkers, such as PD-1, } \\
\text { Intervention (I) }\end{array}$ \\
PD-L1 CTLA-4, IL-6. \\
Any other targeted or non-targeted \\
therapy \\
Outcomes (O) & $\begin{array}{l}\text { Health-economic outcomes } \\
\text { (incremental cost-effectiveness ratio, } \\
\text { net health benefit, net monetary } \\
\text { benefit, LYs, QALYs) } \\
\text { Health-economic evaluations } \\
\text { reporting cost-effectiveness analysis, } \\
\text { cost-utility analysis, net-monetary } \\
\text { benefit }\end{array}$ \\
\hline
\end{tabular}

IL-6, interleukin-6; LYs, life years; PD-1, programmed death-1; PDL1, programmed death ligand-1; QALYs, quality adjusted life years.

final revision based on the evidence that emerged. Quality of studies will be assessed using the CHEERS checklist and finally, the quality of the evidence will be graded according to Grading of Recommendations Assessment, Development and Evaluation system, ${ }^{28}$ assessing heterogeneity, consistency and risk of bias.

All studies and their individual elements will be graded in terms of adequacy of the study regarding the research question, risk of selection bias, measurement of exposure and assessment of outcomes. Disagreements will be resolved by third reviewers.

\section{Study registration}

The study is prospectively registered in PROSPERO, the International Prospective Register of Systematic Reviews.

\section{Data description}

This review will systematically describe the extent of available evidences investigating the predictive biomarkers used in immunotherapy and their health-economic impact. The use of biomarkers to monitor the clinical outcome of patients treated with ICIs may help to reduce the incidence of adverse events related to the immune system thus also improving quality of life. Furthermore, from the pharmacoeconomic evaluations already conducted on these immune biomarkers we expect to find that their use is associated with better cost-effectiveness (or cost-utility, net-monetary benefit) ratio due to their improved ability to predict clinical outcome and to redirect non-reactive patients towards alternative and more effective and cost-effective therapeutic approaches.

Accordingly, main strength of the present work will consist in having an overview on what is already know on immune biomarkers use to guide choice and personalisation of treatment for patients with cancer treated with ICIs. Also, we will try to gather considerations about the diffusion of their real use through economic evaluations that report their outcomes in terms of incremental cost-effectiveness ratio or cost-utility ratio and patients' health-related quality of life. So, results expected from the systematic review will strictly depend on the study design used. We aim to consider the study design such as cost-effectiveness analysis, cost-utility analysis, budget impact analysis, highlighting first the methodology used in the study and to report for each biomarker used in patients with cancer their cost-effectiveness, willingness to pay with the reference threshold. online supplemental appendix 2 shows the hypothetical structure of the data synthesis.

Other systematic reviews on biomarkers were already published evaluating cost-related aspects but they are specifically focused on a cancer condition and the pertaining biomarker. ${ }^{29-32}$ Particularly, our study differs to that of Oosterhoff $e t a l^{29}$ as they aimed to widely investigate the methodological characteristics of economic evaluations on biomarkers and examine economic aspect. To the best of our knowledge this is the first systematic review published broadly exploring the health-economic impact of predictive biomarkers specifically used in treatment of solid tumours with ICIs comparing them with other targeted and non-targeted therapeutic strategies that do not include the use of the reference biomarker.

A potential limitation relates to the heterogeneity associated to the study conducted on biomarkers. Accordingly, between-study heterogeneity may not support the conduct of quantitative meta-analysis. Based on the results obtained, any heterogeneity of the studies will be managed by grouping, if feasible, the included records into different classes such as solid tumour type (eg, breast cancer, bladder cancer, cervical cancer, colon cancer, 
head and neck cancer, Hodgkin lymphoma, liver cancer, lung cancer, renal cell cancer, skin cancer, stomach cancer, rectal cancer) and study design type (eg, costeffectiveness analysis, cost-utility analysis, net-monetary benefit). The same variables present in online supplemental appendix 2 will be evaluated for each group and subgroup.

\section{Patient and public involvement \\ No patients involved.}

\section{ETHICS AND DISSEMINATION}

Results of the systematic review will be published in a peer-reviewed journal and disseminated at a range of health research conferences. The systematic review is part of a larger project funded by Progetti di ricerca di Rilevante Interesse Nazionale (PRIN) 2017 whose aims include the identification of biomarkers able to predict immunotherapeutic-related adverse drug reactions and the potential cost-effectiveness and quality of life of personalised therapies based on advanced tools.

Finally, this systematic review will assess the costeffectiveness implications of using biomarkers in patients with cancer treated with ICIs compared with any other target therapy or conventional therapy without the use of biomarkers. This review may help to understand if this approach may be cost-effective in clinical practice and how the customisation of therapy can actually affect a decrease in costs for the healthcare systems.

\section{Author affiliations}

1Department of Pharmacy, University of Naples Federico II, CIRFF, Center of Pharmacoeconomics and Drug Utilization Research, Naples, Italy

${ }^{2}$ Scuola Superiore Sant'Anna, Institute of Management, Pisa, Italy

${ }^{3}$ University Hospital of Pisa, Unit of Clinical Pharmacology and Pharmacogenetics,

Pisa, Italy

${ }^{4}$ Section of Pharmacology 'L. Donatelli', University of Campania 'L. Vanvitelli', Department of Experimental Medicine, Napoli, Italy

Contributors EM designed and conceptualised this review. SM, V0, VL and IT drafted the protocol. All authors were involved in checking various steps of the search strategy, including keywords, as well as the final version of the protocol. SM, VL and IT were involved in the definition of specific criteria for the extraction of information from studies included and in the development of the strategy for the qualitative data analysis. MDR, AC, RD and GT were involved in establishing eligibility criteria and data extraction forms. GT and EM supervised all work stages. $\mathrm{RD}$ was the funding acquisition supervisor. All authors reviewed and agreed the final version of the manuscript.

Funding This research is funded by Ministero dell'Istruzione, dell'Università e della Ricerca (MIUR) in the framework of the PRIN Project 2017, grant number 2017NR7W5K.

Competing interests None declared.

Patient consent for publication Not required.

Provenance and peer review Not commissioned; externally peer reviewed.

Supplemental material This content has been supplied by the author(s). It has not been vetted by BMJ Publishing Group Limited (BMJ) and may not have been peer-reviewed. Any opinions or recommendations discussed are solely those of the author(s) and are not endorsed by BMJ. BMJ disclaims all liability and responsibility arising from any reliance placed on the content. Where the content includes any translated material, BMJ does not warrant the accuracy and reliability of the translations (including but not limited to local regulations, clinical guidelines, terminology, drug names and drug dosages), and is not responsible for any error and/or omissions arising from translation and adaptation or otherwise.

Open access This is an open access article distributed in accordance with the Creative Commons Attribution Non Commercial (CC BY-NC 4.0) license, which permits others to distribute, remix, adapt, build upon this work non-commercially, and license their derivative works on different terms, provided the original work is properly cited, appropriate credit is given, any changes made indicated, and the use is non-commercial. See: http://creativecommons.org/licenses/by-nc/4.0/.

ORCID iD

Sara Mucherino http://orcid.org/0000-0003-3357-5655

\section{REFERENCES}

1 Gibson S, Raziee HR, Lemmens T. Why the shift? taking a closer look at the growing interest in niche markets and personalized medicine. World Med Health Policy 2015;7:3-27.

2 Collier R. Bye, bye blockbusters, hello niche busters. CMAJ 2011;183:E697-8.

3 Kather JN, Halama N, Jaeger D. Genomics and emerging biomarkers for immunotherapy of colorectal cancer. Semin Cancer Biol 2018;52:189-97.

4 Del Re M, van Schaik RHN, Fogli S, et al. Blood-Based PD-L1 analysis in tumor-derived extracellular vesicles: applications for optimal use of anti-PD-1/PD-L1 axis inhibitors. Biochim Biophys Acta Rev Cancer 2021;1875:188463.

5 Emens LA. Breast cancer immunotherapy: facts and hopes. Clin Cancer Res 2018;24:511-20.

6 Aggen DH, Drake CG. Biomarkers for immunotherapy in bladder cancer: a moving target. J Immunother Cancer 2017;5:94.

7 Otter SJ, Chatterjee J, Stewart AJ, et al. The role of biomarkers for the prediction of response to checkpoint immunotherapy and the rationale for the use of checkpoint immunotherapy in cervical cancer. Clin Oncol 2019;31:834-43.

8 Food and Drug Administration (FDA). Table of pharmacogenomic biomarkers in drug labeling. Available: https://www.fda.gov/drugs/ science-and-research-drugs/table-pharmacogenomic-biomarkersdrug-labeling [Accessed on 13 July 2020].

9 Darvin P, Toor SM, Sasidharan Nair V, et al. Immune checkpoint inhibitors: recent progress and potential biomarkers. Exp Mol Med 2018;50:1-11.

10 Cavazza M, Compagni A, Jommi C. I farmaci oncologici associati a test genetico-molecolari: Le nuove dinamiche nella prospettiva di imprese, regolatori E oncologi, 2015. Available: https://www. unibocconi.it/wps/wcm/connect/313d080c-8ad8-4130-b60cb7a1b1b1dce1/Monografia_AIOM.pdf?MOD=AJPERES [Accessed 09 Jul 2020].

11 Vaddepally RK, Kharel P, Pandey R, et al. Review of indications of FDA-approved immune checkpoint inhibitors per NCCN guidelines with the level of evidence. Cancers 2020;12:738.

12 Gibney GT, Weiner LM, Atkins MB. Predictive biomarkers for checkpoint inhibitor-based immunotherapy. Lancet Oncol 2016;17:e542-51.

13 Patel SP, Kurzrock R. Pd-L1 expression as a predictive biomarker in cancer immunotherapy. Mol Cancer Ther 2015;14:847-56.

14 Gnjatic S, Bronte V, Brunet LR, et al. Identifying baseline immunerelated biomarkers to predict clinical outcome of immunotherapy. $J$ Immunother Cancer 2017;5:44.

15 Teng $F$, Meng X, Kong L, et al. Progress and challenges of predictive biomarkers of anti PD-1/PD-L1 immunotherapy: a systematic review. Cancer Lett 2018;414:166-73.

16 Brahmer JR, Tykodi SS, Chow LQM, et al. Safety and activity of anti-PD-L1 antibody in patients with advanced cancer. N Engl J Med 2012;366:2455-65.

17 Wu X, Gu Z, Chen Y, et al. Application of PD-1 blockade in cancer immunotherapy. Comput Struct Biotechnol J 2019;17:661-74.

18 Ohaegbulam KC, Assal A, Lazar-Molnar E, et al. Human cancer immunotherapy with antibodies to the PD-1 and PD-L1 pathway. Trends Mol Med 2015;21:24-33.

19 Italian Medicines Agency (AIFA). Banca Dati AIFA. Available: https:// farmaci.agenziafarmaco.gov.it/bancadatifarmaci/cerca-per-principioattivo?princ_att=Nivolumab [Accessed 09 Dec 2020].

20 Italian Medicines Agency (AIFA). Banca Dati AIFA. Available: https:// farmaci.agenziafarmaco.gov.it/bancadatifarmaci/cerca-per-principioattivo?princ_att=Pembrolizumab [Accessed 09 Dec 2020].

21 Italian Medicines Agency (AIFA). Banca Dati AIFA. Available: https:// farmaci.agenziafarmaco.gov.it/bancadatifarmaci/cerca-per-principioattivo?princ_att=Atezolizumab [Accessed 09 Dec 2020]. 
22 Aguiar PN, Perry LA, Penny-Dimri J, et al. The effect of PD-L1 testing on the cost-effectiveness and economic impact of immune checkpoint inhibitors for the second-line treatment of NSCLC. Ann Oncol 2017;28:2256-63.

23 Tartari F, Santoni M, Burattini L, et al. Economic sustainability of anti-PD-1 agents nivolumab and pembrolizumab in cancer patients: recent insights and future challenges. Cancer Treat Rev 2016;48:20-4.

24 Georgieva M, da Silveira Nogueira Lima JP, Aguiar P, et al. CostEffectiveness of pembrolizumab as first-line therapy for advanced non-small cell lung cancer. Lung Cancer 2018;124:248-54.

25 Moher D, Shamseer L, Clarke M, et al. Preferred reporting items for systematic review and meta-analysis protocols (PRISMA-P) 2015 statement. Syst Rev 2015;4:1.

26 Page MJ, McKenzie JE, Bossuyt PM, et al. The PRISMA 2020 statement: an updated guideline for reporting systematic reviews. $J$ Clin Epidemiol 2021;134:178-89.

27 Husereau D, Drummond M, Petrou S, et al. Consolidated Health Economic Evaluation Reporting Standards (CHEERS)--explanation and elaboration: a report of the ISPOR Health Economic Evaluation Publication Guidelines Good Reporting Practices Task Force. Value Health 2013;16:231-50.

28 Balshem H, Helfand M, Schünemann HJ, et al. Grade guidelines: 3. rating the quality of evidence. J Clin Epidemiol 2011;64:401-6.

29 Oosterhoff M, van der Maas ME, Steuten LMG. A systematic review of health economic evaluations of diagnostic biomarkers. Appl Health Econ Health Policy 2016;14:51-65.

30 Klein SJ, Brandtner AK, Lehner GF, et al. Biomarkers for prediction of renal replacement therapy in acute kidney injury: a systematic review and meta-analysis. Intensive Care Med 2018;44:323-36.

31 Seo MK, Cairns J. Do cancer biomarkers make targeted therapies cost-effective? A systematic review in metastatic colorectal cancer. PLoS One 2018;13:e0204496.

32 Pathak A, Pandey SP, Madhukar P, et al. Blood biomarkers for the differentiation of cardiac ischemic stroke subtypes: a systematic review. Cardiovasc Hematol Disord Drug Targets 2019;19:215-27. 